\title{
Tenorrafia con ancla de 1.3-mm en zona I del tendón extensor de la mano: evolución funcional a los 6 meses
}

\author{
Tenorraphy with 1.3-mm anchor in zone I of the extensor tendon \\ of the hand: functional evolution at 6 months
}

\author{
Francisco García-Lira, * Jorge H Arce-González, ${ }^{\ddagger}$ Rafael Ortega-Orozco, \\ Raquel A Montes-Pérez," Salvador Magallón-Jaimes"l
}

\begin{abstract}
*Especialista en Traumatología y Ortopedia. Alta Especialidad en Cirugía de Mano. Jefe de la Clínica de Mano en Medyarthros Artroscopia y Medicina Deportiva; ‡Especialista en Traumatología y Ortopedia. Alta Especialidad en Artroscopia y Medicina Deportiva. Alta Especialidad en Cirugía de Mano; Especialista en Traumatología y Ortopedia. Alta Especialidad en Artroscopia y Medicina Deportiva. Director de Medyarthros Artroscopia y Medicina Deportiva. Jefe de la Clínica de Artroscopia y Medicina Deportiva;" Especialista en Traumatología y Ortopedia. Alta Especialidad en Cirugía de Mano. Adjunta a la Clínica de Mano en Medyarthros Artroscopia y Medicina Deportiva; "Especialista en Traumatología y Ortopedia. Capacitación en Alta especialidad en Cirugía de Mano. Adjunto a la Clínica de Mano en Medyarthros Artroscopia y Medicina Deportiva.
\end{abstract}

Guadalajara, Jalisco, México.

\begin{abstract}
Resumen
Introducción: Las lesiones de los tendones extensores en la mano son frecuentes en la práctica diaria del cirujano ortopédico, en la actualidad la zona I extensora de la mano, no tiene un estándar de oro de tratamiento. Objetivo: Describir los resultados funcionales de la reparación quirúrgica, con el uso de anclas de 1.3- $\mathrm{mm}$, de rupturas del tendón extensor de la mano en la zona I, y seguimiento a los seis meses de evolución postquirúrgica. Material y métodos: Se incluyó una cohorte de forma retrospectiva de pacientes sometidos a tenorrafia con anclas de 1.3-mm (MICROFIX QUICKANCHOR Plus DePuy MitekTM Raynham, MA) del tendón extensor de la mano en la zona I por lesiones traumáticas y seguimiento a seis meses después de la cirugía. Los pacientes fueron evaluados desde marzo de 2013 hasta febrero de 2017. Los grados de movilidad, flexión y extensión de la articulación interfalángica distal (DIP) se agruparon en cuatro categorías: mala, regular, buena y excelente. Resultados: Se incluyeron 43 pacientes con ruptura del tendón extensor de la mano en la zona I que fueron tratados con tenorrafia con ancla de 1.3- $\mathrm{mm}$. La extensión de la articulación DIP fue buena en 34 casos (79\%) y regular en nueve (21\%); la flexión fue excelente en 23 casos (54\%) y buena en 15 (35\%). Conclusión: La tenorrafia con ancla de 1.3-mm es un procedimiento novedoso que se ha descrito por primera vez para el tratamiento de la ruptura del tendón extensor de la mano en la zona I. Los resultados funcionales de la articulación DIP seis meses después del procedimiento fueron superiores a los reportados con las técnicas convencionales.
\end{abstract}

Palabras clave: Lesión en zona extensora, tenorrafia con ancla de $1.3 \mathrm{~mm}$, arcos de movimiento.

\begin{abstract}
Introduction: Injuries to the extensor tendons in the hand are common in the daily practice of the orthopedic surgeon. Currently, the extensor zone I of the hand does not have a gold standard of treatment. Objective: To describe the functional results of the surgical repair, with a 1.3-mm anchor, of rupture of the extensor tendon in zone I of the hand and follow-up at six months post-surgical evolution. Material and methods: We included a retrospective cohort of patients subjected to tenorrhaphy with a 1.3-mm anchor (MICROFIX QUICKANCHOR Plus DePuy Mitek ${ }^{T M}$ Raynham, MA) of the extensor tendon in zone I of the hand with traumatic lesions and follow-up six months after surgery. The patients were evaluated from March 2013 to February 2017. The degrees of mobility, flexion and extension of the distal interphalangeal (DIP) joint were grouped into four categories: poor, fair, good and excellent. Results: We included 43 patients with extensor tendon rupture in zone I of the hand who were managed with tenorrhaphy with a 1.3-mm anchor. Extension of the DIP joint was good in 34 (79\%) cases and was regular in 9 (21\%); flexion was excellent in 23 (54\%) cases and good in 15 (35\%). Conclusion: Tenorrhaphy with a 1.3- $\mathrm{mm}$ anchor is a novel procedure that has been described for the first time for the management of
\end{abstract}

Correspondencia:

Dr. Francisco García-Lira

E-mail: drfcogarcialira@ hotmail.com

Recibido: 10-04-2021. Aceptado: 13-04-2021.
Citar como: García-Lira F, Arce-González JH, Ortega-Orozco R, Montes-Pérez RA, Magallón-Jaimes S. Tenorrafia con ancla de 1.3-mm en zona I del tendón extensor de la mano: evolución funcional a los 6 meses. Orthotips. 2021; 17 (2): 69-74. https://dx.doi.org/10.35366/99899 
extensor tendon rupture in zone I of the hand. The functional results of the DIP joint, six months after the procedure, were superior to those reported with conventional techniques.

Keywords: Extensor zone injury, tenorraphy with 1.3-mm anchor, arches of motion.

\section{Introducción}

Las lesiones de los tendones extensores de la mano son frecuentes en la práctica diaria del cirujano ortopédico; sin embargo, en muchos casos están infravalorados en comparación con las lesiones de los tendones flexores. ${ }^{1-7}$ La ruptura de los tendones extensores es más frecuente en el dedo medio, sobre todo en pacientes jóvenes; por el contrario, esta lesión es rara en el pulgar. ${ }^{8,9}$

El mecanismo flexor y extensor ayuda a proporcionar un equilibrio preciso de la fuerza y la posición de los dedos; por tanto, es necesaria una reparación óptima del tendón extensor para evitar secuelas funcionales (Figura 1). El tratamiento de la ruptura de los tendones extensores en la zona I de la mano es controvertido: incluye fijación estática e inmovilización durante seis semanas; recientemente, se ha preferido la reparación quirúrgica (tenorrafia) para restaurar la biomecánica y obtener mejores resultados funcionales (Figura 2). Además, el uso de anclaje óseo, específicamente con un anclaje de 1.3-mm, ofrece una fijación ósea adecuada y una mayor estabilidad para la reparación del tendón. ${ }^{5,10-12}$ En un estudio experimental de cadáveres, se demostró el uso de un ancla para el tratamiento de la ruptura del tendón extensor en la zona I de la mano, con mínimas complicaciones; ${ }^{13}$ sin embargo, no se ha descrito la evolución y complicaciones en pacientes con ruptura del tendón extensor manejados con tenorrafia con anclaje de 1.3-mm en la zona extensora I de la mano. El objetivo de nuestro estudio fue describir los resultados funcionales de la reparación quirúrgica, con el uso de un ancla de 1.3-mm, de la ruptura del tendón extensor en la zona I a los seis meses de evolución postquirúrgica. Nuestra hipótesis es que los resultados funcionales son mejores con el uso de un ancla de 1.3-mm para la fijación distal del tendón extensor.

\section{Material y métodos}

Se incluyó una cohorte retrospectiva de pacientes de ambos sexos, con edades comprendidas entre $18 \mathrm{y}$
65 años, sometidos a tenorrafia con un anclaje de 1.3mm (MICROFIX QUICKANCHOR Plus DePuy Mitek ${ }^{\mathrm{TM}}$ Raynham, MA) por lesión traumática aguda del tendón extensor de la mano en la zona I (Figura 3). El periodo de estudio fue de marzo de 2013 a febrero de 2017 en la Clínica Medyarthros de la ciudad de Guadalajara, Jalisco, México. Los pacientes que cumplieron con el protocolo de rehabilitación indicado y que aceptaron participar fueron incluidos en el estudio; no se incluyeron pacientes con lesiones en áreas distintas a la zona extensora I de la mano o con lesión ósea asociada y/o lesiones expuestas. El criterio de exclusión fue el incumplimiento de las visitas posteriores a la tenorrafia y el tabaquismo durante el proceso de curación. Todos los pacientes dieron su consentimiento informado para participar. El estudio fue aprobado por el Comité Institucional de Ética e Investigación.

Se evaluaron las siguientes variables: edad en años, sexo, actividad laboral, destreza manual, dedo lesionado, mecanismo de lesión y grados de movilidad en flexión y extensión de la articulación interfalángica distal (DIP) a los seis meses de la cirugía. En la literatura no existe una interpretación cualitativa de los rangos de flexión-extensión para evaluar la recuperación funcional después de la tenorrafia del ligamento extensor de la mano en la zona I. Por tanto, la flexión y la extensión se clasificaron arbitrariamente en cuatro categorías: mala, regular,

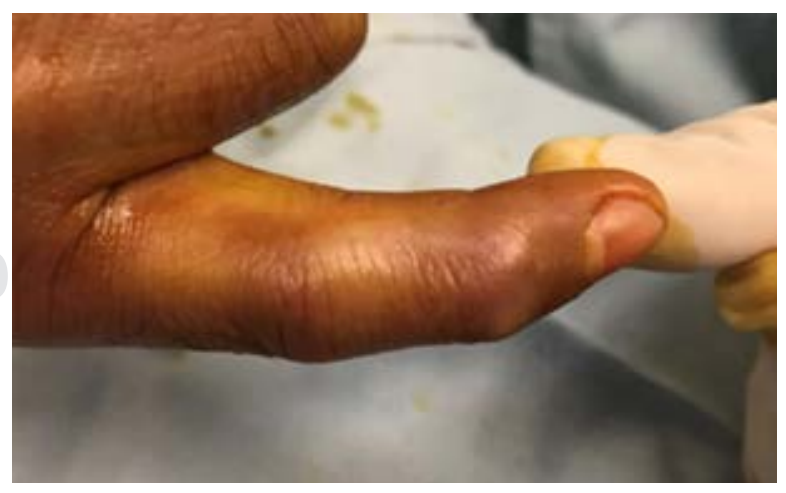

Figura 1: Articulación interfalángica distal en flexión, por ruptura del tendón extensor en la zona I del meñique de la mano derecha. 


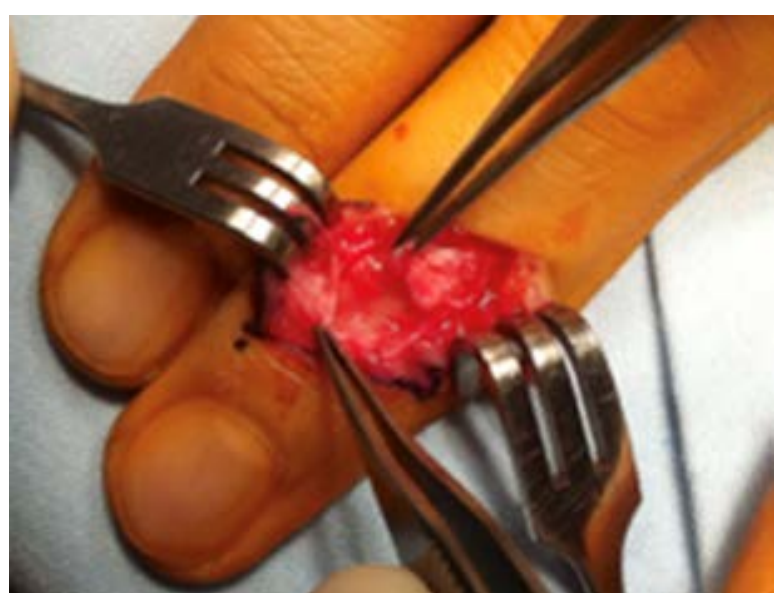

Figura 2: Ruptura del tendón extensor en la zona I del dedo medio de la mano derecha.

buena y excelente. El protocolo de diagnóstico por imagen utilizado incluyó radiografías anteroposteriores y oblicua, y resonancia magnética nuclear (1.5 Tesla) de la mano.

\section{Técnica quirúrgica}

Se realizó un miniabordaje dorsal del dedo en la zona I extensora de la mano. Una vez localizados los polos, proximal y distal, se procedió a desbridarlos y fijar la articulación en extensión con un clavo de Kirschner de $1.8 \mathrm{~mm}$ (Figura 4). Después se colocó un ancla de 1.3-mm en la falange distal bajo visión fluoroscópica, seguida de tenorrafia (Figura 5). El dedo operado se protegió con una férula e inmovilización, incluido el dedo adyacente que se eligió para mantener la mayor funcionalidad de la mano. La sutura superficial se retiró dos semanas después del procedimiento quirúrgico, mientas que el clavo Kirshner y la férula se retiraron cuatro semanas después de la cirugía.

\section{Análisis estadístico}

Se utilizó estadística descriptiva con frecuencias y proporciones. La información se registró en una base de datos Excel (Microsoft ${ }^{\mathrm{TM}}$ Seattle, WA, EE. UU.).

\section{Resultados}

Se incluyeron 43 pacientes con ruptura del tendón extensor de la mano en la zona I a los que se les reali- zó tenorrafia con ancla de 1.3-mm: 28 (65\%) hombres y 15 (35\%) mujeres, con una edad media de 36 años (Tabla 1). No se eliminó ningún caso, ya que todos los pacientes completaron las visitas de seguimiento. En el grupo de estudio, las actividades más frecuentes fueron las siguientes: actividades domésticas $(n=11$, $25.58 \%$ ), oficinistas $(n=11,25.58 \%$ ) y deportistas de alto rendimiento $(n=7,16.27 \%)$. La mano derecha fue la más afectada ( $n=35,81.39 \%)$, y los dedos índice y medio fueron los más lesionados ( $n=13,30 \%$ vs $\mathrm{n}=15,35 \%$ respectivamente). El traumatismo directo con el balón ( $n=12,28 \%)$ fue el principal mecanismo de ruptura del tendón extensor.

El seguimiento se realizó durante seis meses después de la tenorrafia; los rangos de movimiento de la articulación DIP para determinar la recuperación funcional del dedo lesionado se muestran en la Tabla 2. En extensión, se obtuvieron buenos resultados en 34 casos $(79 \%)$, con rango de movimiento mayor a $-9^{\circ}$ (promedio $-1^{\circ}$ ), mientras que nueve $(21 \%)$ mostraron resultados regulares, con rango de movimiento entre $-29^{\circ}$ a $-10^{\circ}$ (promedio $-12^{\circ}$ ). En la evaluación de la flexión, $23(54 \%)$ pacientes recuperaron movimiento mayor a $31^{\circ}$ con excelentes resultados (Tabla 2 ); en 15 casos (35\%) se obtuvieron buenos resultados (promedio de $29^{\circ}$ ), mientras que cinco pacientes (11\%) presentaron resultados regulares (promedio de 19\%); no se encontraron malos resultados en extensión o flexión en ningún paciente.

No se produjeron complicaciones transquirúrgicas en los pacientes incluidos en el estudio; en el seguimiento, un paciente desarrolló un absceso en la primera semana de evolución que fue tratado con

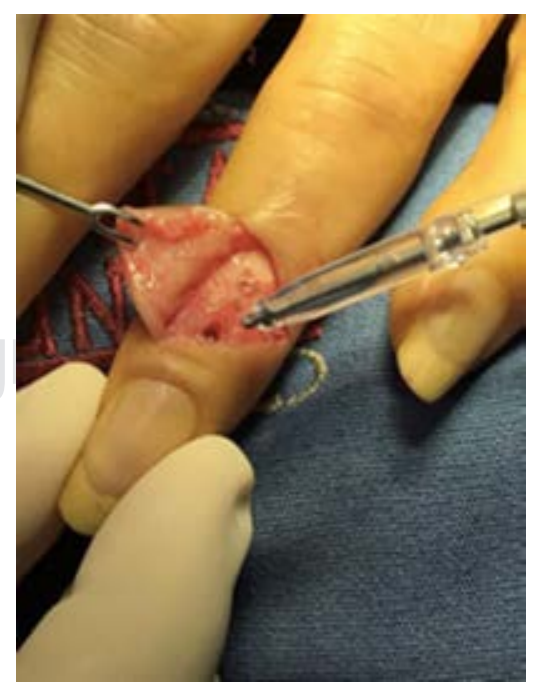

Figura 3:

Ancla Mitek ${ }^{\mathrm{TM}}$ de 1.3-mm. 


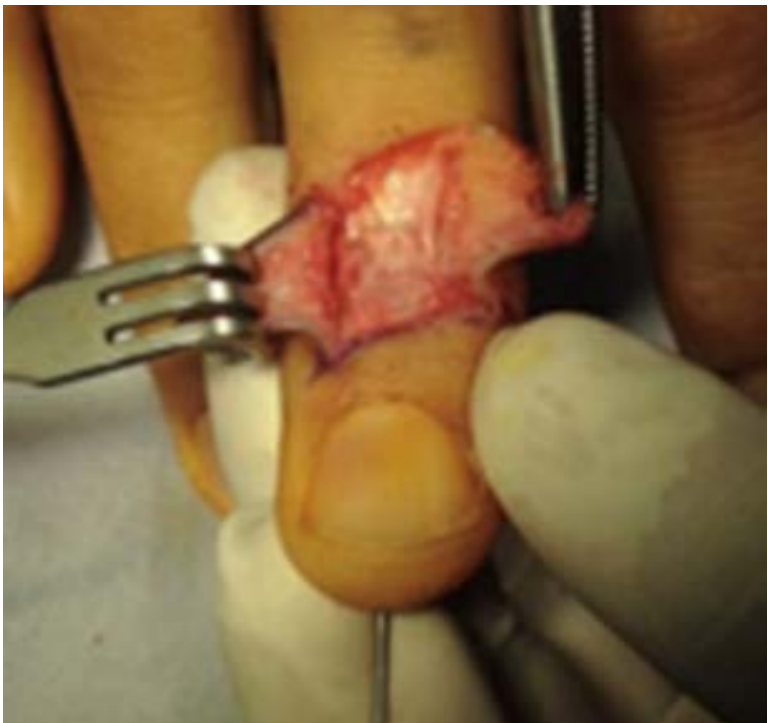

Figura 4: Fijación con un clavo Kirschner de $1.8 \mathrm{~mm}$ de la articulación interfalángica distal para limitar la flexión-extensión para proteger la tenorrafia y lograr una reparación exitosa del tendón.

drenaje y antibióticos, con evolución satisfactoria. Sin embargo, se presentó un granuloma en el lecho quirúrgico cuatro meses después de la cirugía; se realizó desbridamiento quirúrgico, con recuperación favorable. Seis meses después de la operación, un paciente presentó rigidez de la articulación DIP que no se recuperó.

\section{Discusión}

En nuestro estudio se demostraron excelentes resultados funcionales en flexión y buena recuperación en extensión con tenorrafia con ancla de 1.3-mm para el tratamiento de la ruptura del tendón extensor de la mano en la zona I. Hasta donde sabemos, nuestro estudio es el primero en el mundo en demostrar que la cirugía que emplea esta técnica es efectiva, con resultados superiores a los reportados con técnicas convencionales.

Se han mostrado buenos resultados con tenorrafia utilizando diferentes técnicas quirúrgicas en las áreas extensoras III a $\mathrm{V}$ de la mano, mientras que los resultados en la zona I y II han sido malos, principalmente porque estas zonas incluyen tendones delgados y superficiales con malos resultados funcionales por disminución de la extensión ( 11 a $45^{\circ}$ ) y flexión (21 a $45^{\circ}$ ) tras la reparación quirúrgica. $6,10,11,14-18$ En un estudio ${ }^{13}$ de 24 dedos de seis cadáveres, se evaluaron tres técnicas quirúrgicas diferentes para la reparación del tendón extensor de la mano en la zona I: banda de tensión, extracción y uso de ancla; los resultados de la fuerza del tendón extensor fueron similares entre los tres métodos, mientras que el procedimiento con ancla, que funciona como un implante intraóseo, arrojó mejores resultados en los tejidos blandos, con menor exposición de las suturas. En nuestro estudio se incluyeron 43 pacientes con el mismo número de dedos lesionados; los resultados funcionales en extensión mostraron una media de $-1^{\circ}$ y flexión superior a $31^{\circ}$ en más de la mitad de los pacientes. Su recuperación funcional fue mejor que la reportada para otras técnicas. ${ }^{13} \mathrm{El}$ anclaje óseo es un procedimiento eficaz para proporcionar la fijación del tendón al plano esquelético; esta forma de reparación de la ruptura del tendón extensor de la zona I de la mano es fácil de realizar, es sencilla y no requiere de implantes adicionales que puedan dañar los tejidos blandos circundantes. ${ }^{12}$ Una vez colocado el anclaje, se fija en el hueso, y la simple unión de las suturas completa la fijación. Un beneficio adicional del ancla es que no requiere una segunda intervención para su remoción.

Se utilizó una clasificación, a criterio de los autores, para describir el rango de movimiento de la articulación DIP, con las categorías de malo, regular, bueno y excelente en el periodo postoperatorio. Esta clasificación debe validarse en estudios posteriores que incluyan mediciones en los periodos pre y postquirúrgico. Las principales complicaciones de la reparación del tendón extensor son las adherencias, en especial en las zonas I a III; otras complicaciones incluyen infección, granuloma, exposición del implante

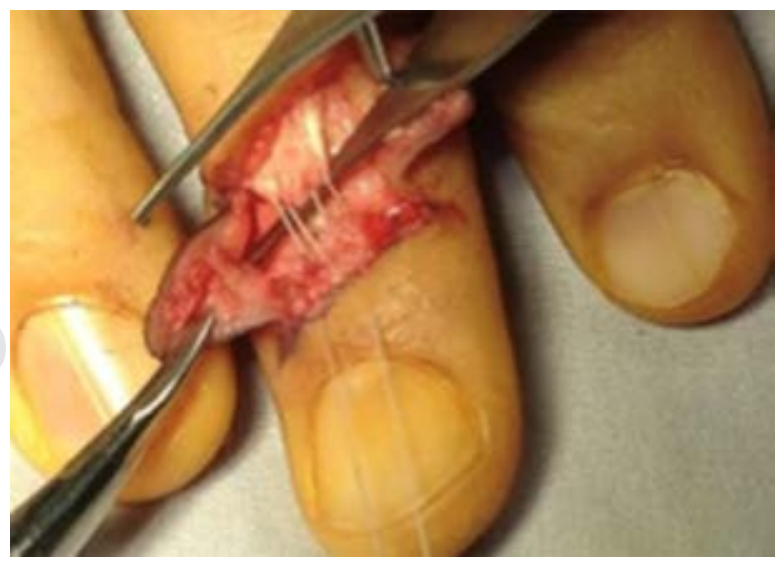

Figura 5: Sutura con ancla Mitek ${ }^{\mathrm{TM}}$ de 1.3-mm con inserción en la falange distal. 
Tabla 1: Población de estudio y características de la lesión del tendón extensor de la mano en la zona I ( $\mathrm{N}=43$ ).

\begin{tabular}{llc}
\hline & & \\
& & $\mathrm{n}(\%)$ \\
\hline Sexo & Mujeres & $15(35)$ \\
& Hombres & $28(65)$ \\
Edad & Promedio & 36 \\
& Rango & $20-67$ \\
Actividad & Actividades domésticas & $11(26)$ \\
de trabajo & Estudiantes & $5(12)$ \\
& Trabajadores de ciencias de la salud & $3(7)$ \\
& Empresarios & $4(9)$ \\
& Trabajadores de oficina & $11(26)$ \\
& Deportistas de alto rendimiento & $7(16)$ \\
& Otros & $2(5)$ \\
Lado & Derecho & $35(81)$ \\
afectado & Izquierdo & $8(19)$ \\
Dedo & Dedo índice & $13(30)$ \\
lesionado & Dedo medio & $15(35)$ \\
& Dedo anular & $5(12)$ \\
& Dedo meñique & $10(23)$ \\
& Pulgar & $0(0)$ \\
Mecanismo & Aplastamiento & $4(9)$ \\
de lesión & Trauma directo con pelota & $12(28)$ \\
& Trauma directo con otros objetos & $9(21)$ \\
& Caída de propia altura & $8(19)$ \\
& Desconocido & $2(5)$ \\
& Lesión con cuchillos, mesa o pared & $8(19)$ \\
\hline & &
\end{tabular}

y rigidez articular. ${ }^{16,18,19}$ Los factores relacionados con las complicaciones son diversos. En nuestro estudio, en tres pacientes (7\%) las complicaciones incluyeron infección (un caso), granuloma (un caso) y rigidez articular (un caso). Por el contrario, en un estudio con 24 dedos de cadáver con técnica de extracción o banda de tensión, se informó un mayor número de complicaciones, incluida la exposición del implante; el uso de anclajes no produjo esta complicación. ${ }^{13}$ Del mismo modo, en nuestra investigación no hubo casos con exposición del implante. Un beneficio potencial de la tenorrafia con el uso de un ancla es la ausencia de adherencias postoperatorias, como se demostró en nuestra publicación; hay menos movilidad asociada con esta técnica novedosa.

Los puntos fuertes del estudio están relacionados con una innovadora técnica quirúrgica para la reparación del tendón extensor en la zona I de la mano que fueron intervenidos por el mismo cirujano (Francisco García Lira), y se incluyó un número suficiente de pacientes para demostrar la eficacia del procedimiento. Las limitaciones del estudio están relacionadas con el diseño retrospectivo: los rangos de movimiento de la articulación DIP no fueron evaluados antes de la intervención quirúrgica. Asimismo, el mecanismo de lesión del tendón en la mayoría de los casos incluidos estuvo relacionado con el traumatismo deportivo; por tanto, debe evaluarse la eficacia de la tenorrafia con ancla de 1.3-mm en lesiones por otros mecanismos. Cabe señalar que el alto costo del implante limita su uso generalizado.

\section{Conclusiones}

En nuestro estudio, la eficacia de la tenorrafia se demostró con el uso de un ancla de 1.3-mm en la ruptura del tendón extensor de la mano en la zona I, con excelentes resultados funcionales en flexión y buena recuperación en extensión; esta novedosa técnica quirúrgica tiene las siguientes características: 1) proporciona una fijación sólida; 2) reduce la exposición de los tejidos blandos; 3) disminuye el periodo trans y postoperatorio; y 4) acorta el tiempo de uso del manguito hemostático. La ruptura del tendón extensor en la zona I de la mano ocurre con frecuencia en nuestra población y el manejo inadecuado causa deformidad crónica y limitación funcional. Es necesario un conocimiento profundo de la anatomía del mecanismo extensor y las posibles complicaciones. La técnica descrita es sencilla y los resultados fun-

Tabla 2: Rango de movimiento de la articulación interfalángica distal del dedo lastimado ( $\mathrm{N}=43$ ).

\begin{tabular}{|c|c|c|c|c|c|c|c|}
\hline Extensión & $\mathrm{n}$ & Grados de movimiento: promedio & Escala cualitativas & Flexión & $n$ & Grados de movimiento: promedio & Escala cualitativa* \\
\hline$<-30^{\circ}$ & 0 & - & Mala & $0-10^{\circ}$ & 0 & - & Mala \\
\hline$-29^{\circ} \mathrm{a}-10^{\circ}$ & 9 & $-12^{\circ}$ & Regular & $11-20^{\circ}$ & 5 & $19^{\circ}$ & Regular \\
\hline \multirow[t]{2}{*}{$-9^{\circ} 0>^{*}$} & 34 & $-1^{0}$ & Buena & $21-30^{\circ}$ & 15 & $29^{\circ}$ & Buena \\
\hline & & & & $>31^{\circ}$ & 23 & $37^{\circ}$ & Excelente \\
\hline
\end{tabular}

$\S$ Flexión y extensión fueron clasificados arbitrariamente en cuatro categorías: mala, regular, buena y excelente.

* $>$ Los grados de extensión se muestran con tendencia a la positividad. 
cionales satisfactorios. Por tanto, en la opinión de los autores, este método puede ser el tratamiento de elección para la ruptura del tendón extensor de la mano en la zona I.

\section{Agradecimientos}

Dra. Ana María Contreras, de la Unidad de Investigación y Desarrollo Tecnológico Medyarthros (UNIDET) por su asesoramiento en la redacción del artículo.

\section{Referencias}

1. Crosby CA, Wehbé MA. Early protected motion after extensor tendon repair. J Hand Surg Am. 1999; 24 (5): 1061-1070.

2. Hauge MF. The results of tendon suture of the hand; a review of 500 patients. Acta Orthop Scand. 1955; 24 (3): 258-270.

3. Boyer MI, Chang J. Part XVII. Tendon: traumatic. Extensor tendon laceration. 100 hand cases. New York: Thieme Medical Publishers Inc; 2016. pp. 209-210.

4. Utrillas Compaired A, Corella Montoya F, Delgado Martínez AD. Lesiones agudas de tendones extensores de la mano. En: Delgado Martínez AD, editor. Cirugía ortopédica y traumatología. 2a edición. Madrid, España: Editorial Médica Panamericana; 2012. pp. 503-507.

5. Merle M, Lim A, Jager $\mathrm{T}$, et al. Injuries of the extensor apparatus. In: Merle M, Dautel G. Emergency surgery of the hand. Philadelphia: Elsevier Masson SAS; 2017. pp. 303-325.

6. Tuncali D, Yavuz N, Terzioglu A, Aslan G. The rate of upperextremity deep-structure injuries through small penetrating lacerations. Ann Last Surg. 2005; 55: 146-148.

7. Chao JD, Sarwahi V, Da Silva YSS, Rosenwasser MP, Strauch RJ. Central slip tenotomy for the treatment of chronic mallet finger: an anatomic study. J Hand Surg Am. 2004; 29A (2): 216-219.

8. McMurty JT, Isaacs J. Extensor tendons injuries. Clin Sports Med. 2015; 34 (1): 167-180.
9. Patillo D, Rayan GM. Open extensor tendon injuries: an epidemiologic study. J Hand Surg Am. 2012; 17 (1): 37-42.

10. Colzani G, Tos P, Battiston B, Merolla G, Porcellini G, Artiaco $S$. Traumatic extensor tendon injuries to the hand: clinical anatomy, biomechanics, and surgical procedure review. J Hand Microsurg. 2016; 8: 2-12.

11. Bickel B, Shapiro DB, Keith MW, et al. Manejo de las lesiones del tendón extensor. En: Chung KC. Reconstrucción de mano y extremidad superior. Filadelfia: Amolca, Actualidades Médicas, C.A; 2011. pp. 47-60.

12. Daluiski A, Tan Virak. Use of suture anchor in hand surgery. Atlas of minimally invasive hand and wrist surgery. New York: Informa healthcare; 2008. pp. 5-9.

13. Cheung JPY, Fung B, Ip WY. Peak load resistance of the Juggerknot ${ }^{\mathrm{TM}}$ soft anchor technique compared with other common fixation techniques for large mallet finger fractures. J Hand Surg Am. 2013; 18 (3): 381-388.

14. Mehdinasab SA, Pipeladeh MR. Results of primary extensor tendon repair of the hand with respect to the zone of injury. Arch Trauma Res. 2012; 1 (3): 131-134.

15. Arora R, Lutz M, Gabl M, Pechlaner S. Primary treatment of acute extensor tendon injuries of the hand. Oper Orthop Traumatol. 2008; 20 (1): 13-24.

16. Griffin M, Hindocha S, Jordan D, Saleh M, Khan W. Management of extensor tendon injuries. Open Orthop J. 2012; 6: 36-42.

17. Purcell T, Nadie PA, Murugan S, O,Donnell M, Lawless M. Static splinting of extensor tendon injuries repairs. J Hand Surg Br. 2000; 25 (2): 180-182.

18. Strauch RJ. Extensor tendon injury. In: Wolfe SW, Hotchkiss RN, Pederson WC, et al. Green's operative hand surgery. 7th edition. Philadelphia: Elsevier, Inc.; 2017. pp. 151-182.

19. Pechlaner S, Kerschbaumer F, Hussl H. Extensor tendon injuries. Atlas of hand surgery. New York: Thieme Stuttgart; 2000. pp. 189-222.

\section{Conflicto de intereses}

Los autores declaran que no existe conflicto de intereses qué revelar. 